\title{
CLINICAL AND IMAGING PRESENTATION OF MOLECULAR TYPES OF BREAST CANCER
}

Vanessa Monteiro Sanvido¹, Morgana Domingues da Silva¹, Patricia Zaideman Charf¹, Simone Elias¹, Afonso Celso Pinto Nazário ${ }^{1}$

'Universidade Federal de São Paulo - São Paulo (SP), Brazil.

Introduction: Breast cancer is the second most common malignant neoplasm among women in Brazil and worldwide. Its incidence increases with age, especially in individuals older than 50 years. Mammography is the main screening test, has high sensitivity, and is the only method that has made an impact on mortality rate. Breast cancer is classified into molecular subtypes, based on immunohistochemical markers. The luminal A subtype (LA) presents estrogen receptor (ER) and progesterone receptor (PR) positive, HER2 negative, and low Ki-67 index. Luminal B (LB) shows ER and/or PR positive, HER2 negative, high Ki-67 index, or HER2 positive (luminal HER2). HER2 has HER2 overexpression and ER and PR negative. Triple-negative (TN) has ER, PR, and HER2 negative and high histological grade. Objective: To evaluate patient characteristics according to the molecular subtypes of breast carcinoma among individuals treated at the Hospital São Paulo - Universidade Federal de São Paulo. Method: This is a retrospective study based on the analysis of medical records of breast cancer cases from the Hospital São Paulo between 2013 and 2016. During this period, 235 patients were treated. Among them, $40 \%$ were classified as LA, $34 \%$ as LB, $8 \%$ as luminal HER2, $15 \%$ as TN, and $3 \%$ as HER2. The mean age was 57.6 years. The incidence of breast carcinoma was higher in women over 50 years of age in all subtypes: $75.2 \%$ for LA, $65 \%$ for LB, 58\% for luminal HER2, 100\% for HER2 overexpression, and 75.1\% for TN. Regarding ethnicity, most women were white in all subtypes, accounting for $66.5 \%$ of cases. In all subtypes, the most common clinical complaint was nodule: $86 \%$ for LA, $86 \%$ for LB, $100 \%$ for HER2 overexpression, and $96 \%$ for TN. Among the mammographic findings, nodule was the most frequent in all subtypes. Luminal subtypes presented other findings, such as suspicious calcifications (14\% for LA and $21 \%$ for LB), focal asymmetries ( $14 \%$ for LA and 5\% for LB), and distortions ( $2 \%$ for LA and $3 \%$ for LB). Conclusion: Breast cancer has a higher incidence among Caucasian individuals and those aged 50 to 60 years. The clinical and imaging presentation of tumors is influenced by their molecular subtype: luminal subtypes have a greater diversity of findings and non-palpable lesions, while TN tumors usually manifest as palpable nodules. 